\title{
Qualitative study of a third order rational system of difference equations
}

\author{
Mehmet GÜmüş, RaAfat Abo-Zeid
}

\begin{abstract}
This paper is concerned with the dynamics of positive solutions for a system of rational difference equations of the following form

$$
u_{n+1}=\frac{\alpha u_{n-1}^{2}}{\beta+\gamma v_{n-2}}, v_{n+1}=\frac{\alpha_{1} v_{n-1}^{2}}{\beta_{1}+\gamma_{1} u_{n-2}}, \quad n=0,1, \ldots,
$$

where the parameters $\alpha, \beta, \gamma, \alpha_{1}, \beta_{1}, \gamma_{1}$ and the initial values $u_{-i}, v_{-i} \in$ $(0, \infty), i=0,1,2$. Moreover, the rate of convergence of a solution that converges to the zero equilibrium of the system is discussed. Finally, some numerical examples are given to demonstrate the effectiveness of the results obtained.
\end{abstract}

\section{INTRODUCTION}

Recently, the analysis of equilibrium solutions of various systems of nonlinear difference equations has been one of the main topics in the theory of dynamical systems (see $[2,4,7,8,9,10,11,13,14,17,18,23,24,26,27]$ and the references cited therein). Difference equations and systems of difference equations play an important role in the analysis of mathematical models of many areas such as ecology, population dynamics, statistical problems, number theory, geometry, genetics in biology, economics, psychology, sociology, physics, engineering, economics (see [1, 3, 5, 12, 15, 16, 21, 22]).

In [6], El-Owaidy et al. studied the third order non-linear rational difference equation

$$
x_{n+1}=\frac{b x_{n-1}^{2}}{A+B x_{n-2}}, \quad n=0,1, \ldots,
$$

where the parameters $A, B, b$ and the initial conditions $x_{-2}, x_{-1}, x_{0}$ are arbitrary non-negative real numbers.

Motivated by the aforementioned study, our goal in this paper is to investigate the equilibrium points, the local asymptotic stability of these points,

2020 Mathematics Subject Classification. Primary: 39A20.

Key words and phrases. System of difference equations, equilibrium, positive solutions, invariant subsets.

Full paper. Received 5 June 2020, revised 25 January 2021, accepted 7 February 2021, available online 16 March 2021. 
the global behavior of positive solutions, the existence of unbounded solutions and the existence of the prime two-periodic solutions of the following system

$$
u_{n+1}=\frac{\alpha u_{n-1}^{2}}{\beta+\gamma v_{n-2}}, v_{n+1}=\frac{\alpha_{1} v_{n-1}^{2}}{\beta_{1}+\gamma_{1} u_{n-2}}, n=0,1, \ldots,
$$

where the parameters $\alpha, \beta, \gamma, \alpha_{1}, \beta_{1}, \gamma_{1}$ and the initial values $u_{-2}, u_{-1}, u_{0}$, $v_{-2}, v_{-1}, v_{0}$ are positive real numbers. This paper is naturel extension of the paper [6].

Note that the system (2) can be reduced to the following system of difference equations

$$
x_{n+1}=\frac{r x_{n-1}^{2}}{1+y_{n-2}}, y_{n+1}=\frac{s y_{n-1}^{2}}{1+x_{n-2}}, n=0,1, \ldots
$$

by the change of variables $u_{n}=\left(\frac{\beta_{1}}{\gamma_{1}}\right) x_{n}$ and $v_{n}=\left(\frac{\beta}{\gamma}\right) y_{n}$ with $r=\frac{\alpha \beta_{1}}{\beta \gamma_{1}}$ and $s=\frac{\alpha_{1} \beta}{\beta_{1} \gamma}$.

We shall study the behavior of the solutions of the system (3) rather than system (2). Note that if

$$
r=s \text { and } x_{-i}=y_{-i} \text { for } i=0,1,2,
$$

in system (3), then the system (3) reduces into Eq.(1) which has been studied by El-Owaidy in [6]. In this paper, we exclude the condition (4).

Using Mathematical induction on $n$, we see that if $x_{-i}, y_{-i}$ are positive real numbers for $i \in\{0,1,2\}$ in system (3), then

$$
\min \left\{x_{n}, y_{n}\right\}>0, n \geq-2 \text {. }
$$

To the best of our knowledge, the difference equations system (2) was not dealt with. Therefore, it is meaningful to study their deep results.

\section{Preliminaries}

For the completeness in this paper, we find useful to remind some basic concepts of the difference equations theory as follows:

Let $f_{1}: I_{1}^{3} \times I_{2}^{3} \rightarrow I_{1}$ and $f_{2}: I_{1}^{3} \times I_{2}^{3} \rightarrow I_{2}$ are continuously differentiable functions where $I_{1}, I_{2}$ are intervals of real numbers. For any initial values $\left(x_{-i}, y_{-i}\right) \in I_{1} \times I_{2}$ for $i \in\{0,1,2\}$, the six-dimensional discrete dynamical system

$$
\left.\begin{array}{rl}
x_{n+1} & =f_{1}\left(x_{n}, x_{n-1}, x_{n-2}, y_{n}, y_{n-1}, y_{n-2}\right), \\
y_{n+1} & =f_{2}\left(x_{n}, x_{n-1}, x_{n-2}, y_{n}, y_{n-1}, y_{n-2}\right)
\end{array}\right\}
$$

has a unique solution $\left\{\left(x_{n}, y_{n}\right)\right\}_{n=-2}^{\infty}$.

Definition 1. An equilibrium point of system (5) is a point $(\bar{x}, \bar{y})$ that satisfies

$$
\bar{x}=f_{1}(\bar{x}, \bar{x}, \bar{x}, \bar{y}, \bar{y}, \bar{y}),
$$




$$
\bar{y}=f_{2}(\bar{x}, \bar{x}, \bar{x}, \bar{y}, \bar{y}, \bar{y}) .
$$

Together with the system (5), if we consider the associated vector map $F=\left(f_{1}, x_{n}, x_{n-1}, f_{2}, y_{n}, y_{n-1}\right)$, then the point $(\bar{x}, \bar{y})$ is also called a fixed point of the vector map $F$.

Definition 2. Let $(\bar{x}, \bar{y})$ be an equilibrium point of the map $F$ where $f_{1}$ and $f_{2}$ are continuously differentiable functions at $(\bar{x}, \bar{y})$. The linearized system of (5) about the equilibrium point $(\bar{x}, \bar{y})$ is

$$
X_{n+1}=F\left(X_{n}\right)=B X_{n}, \quad n=0,1, \ldots,
$$

where $X_{n}=\left(x_{n}, x_{n-1}, x_{n-2}, y_{n}, y_{n-1}, y_{n-2}\right)^{T}$ and $B$ is a Jacobian matrix of the system (5) about the equilibrium point $(\bar{x}, \bar{y})$.

Definition 3. Let $\bar{X}$ be a fixed point of the system of difference equations (6). If no eigenvalues of the Jocobian matrix $B$ about $\bar{X}$ has absolute value equal to one, then $\bar{X}$ is called hyperbolic. If there exists an eigenvalue of the Jocobian matrix $J_{F}$ about $\bar{X}$ with absolute value equal to one, then $\bar{X}$ is called non-hyperbolic.

Theorem 1. Let $\bar{X}$ be a fixed point of the system of difference equations (6). If all eigenvalues of the Jocobian matrix $J_{F}$ about $\bar{X}$ lie inside the open unit disk $|\lambda|<1$, then $\bar{X}$ is locally asymtotically stable. If one of them has a modulus greater than one, then $\bar{X}$ is unstable.

Theorem 2 (Routh-Hurwitz criterion). Assume that $X_{n+1}=F\left(X_{n}\right), n=$ $0,1, \ldots$, is a system of difference equations and $\bar{X}$ is a fixed point of $F$, the characteristic polynomial of this system about the equilibrium point $\bar{X}$ is $P(\lambda)=a_{0} \lambda^{n}+a_{1} \lambda^{n-1}+\cdots+a_{n-1} \lambda+a_{n}=0$, with real coefficients and $a_{0}>0$. Then all roots of the polynomial $P(\lambda)$ lie inside the open unit disk $|\lambda|<1$ if and only if $\Delta_{k}>0$ for $k=1,2, \ldots, n$ where $\Delta_{k}$ is the principal minor of order $k$ of the $n \times n$ matrix

$$
\Delta_{n}=\left(\begin{array}{ccccc}
a_{1} & a_{3} & a_{5} & \cdots & 0 \\
a_{0} & a_{2} & a_{4} & \cdots & 0 \\
0 & a_{1} & a_{3} & \cdots & 0 \\
\vdots & \vdots & \vdots & \ddots & \vdots \\
0 & 0 & 0 & \cdots & a_{n}
\end{array}\right) .
$$

For other basic knowledge about difference equations and their systems, the reader is referred to $[19,20,21]$.

\section{Stability Nature of Equilibrium Points}

In this section, we shall state the equilibrium points of system (3) and we will investigate the stability character of these points. One can easily see that the values of the equilibrium points depends on $r$ and $s$.

Lemma 1. We have the following: 
- $\left(\bar{x}_{0}, \bar{y}_{0}\right)=(0,0)$ is always an equilibrium point of system (3) for all values of $r$ and $s$;

- if $r s>1$, then system (3) has the unique positive equilibrium point $\left(\bar{x}_{1}, \bar{y}_{1}\right)=\left(\frac{s+1}{r s-1}, \frac{r+1}{r s-1}\right)$.

Before we investigate local asymptotic stability of the aforementioned equilibrium points, we shall build the corresponding linearized form of the system (3) and consider the following transformation

$$
\left(x_{n}, x_{n-1}, x_{n-2}, y_{n}, y_{n-1}, y_{n-2}\right) \rightarrow\left(f, f_{1}, f_{2}, g, g_{1}, g_{2}\right),
$$

where

$$
f=\frac{r x_{n-1}^{2}}{1+y_{n-2}}, f_{1}=x_{n}, f_{2}=x_{n-1}, g=\frac{s y_{n-1}^{2}}{1+x_{n-2}}, g_{1}=y_{n}, g_{2}=y_{n-1} .
$$

The Jacobian matrix about the fixed point $(\bar{x}, \bar{y})$ under this transformation is as follows

$$
J_{F}(\bar{x}, \bar{y})=\left(\begin{array}{cccccc}
0 & \frac{2 r \bar{x}}{1+\bar{y}} & 0 & 0 & 0 & -\frac{r \bar{x}^{2}}{(1+\bar{y})^{2}} \\
1 & 0 & 0 & 0 & 0 & 0 \\
0 & 1 & 0 & 0 & 0 & 0 \\
0 & 0 & -\frac{s \bar{y}^{2}}{(1+\bar{x})^{2}} & 0 & \frac{2 s \bar{y}}{1+\bar{x}} & 0 \\
0 & 0 & 0 & 1 & 0 & 0 \\
0 & 0 & 0 & 0 & 1 & 0
\end{array}\right),
$$

where $r, s \in(0, \infty)$.

Theorem 3. The zero equilibrium point $\left(\bar{x}_{0}, \bar{y}_{0}\right)$ of system (3) is locally asymptotically stable.

Proof. The linearized system of (3) about the equilibrium point $\left(\bar{x}_{0}, \bar{y}_{0}\right)$ is given by

$$
X_{n+1}=J_{F}(\bar{x}, \bar{y}) X_{n}
$$

where $X_{n}=\left(x_{n}, x_{n-1}, x_{n-2}, y_{n}, y_{n-1}, y_{n-2}\right)^{T}$ and

$$
J_{F}\left(\bar{x}_{0}, \bar{y}_{0}\right)=\left(\begin{array}{cccccc}
0 & 0 & 0 & 0 & 0 & 0 \\
1 & 0 & 0 & 0 & 0 & 0 \\
0 & 1 & 0 & 0 & 0 & 0 \\
0 & 0 & 0 & 0 & 0 & 0 \\
0 & 0 & 0 & 1 & 0 & 0 \\
0 & 0 & 0 & 0 & 1 & 0
\end{array}\right)
$$

The characteristic equation associated with $J_{F}\left(\bar{x}_{0}, \bar{y}_{0}\right)$ is

$$
P(\lambda)=\lambda^{6}=0 \text {. }
$$

All roots of $P(\lambda)$ are equal to zero. Since all eigenvalues of the Jacobian matrix $J_{F}$ about $(0,0)$ lie inside the open unit disk $|\lambda|<1$, the zero equilibrium point is locally asymptotically stable and this completes the proof. 
Theorem 4. If rs $>1$, then the positive equilibrium point $\left(\bar{x}_{1}, \bar{y}_{1}\right)$ is unstable, namely saddle point.

Proof. The linearized system of (3) about the equilibrium point $\left(\bar{x}_{1}, \bar{y}_{1}\right)=$ $\left(\frac{s+1}{r s-1}, \frac{r+1}{r s-1}\right)$ is given by

$$
X_{n+1}=J_{F}\left(\bar{x}_{1}, \bar{y}_{1}\right) X_{n}, n=0,1, \ldots,
$$

where $X_{n}=\left(x_{n}, x_{n-1}, x_{n-2}, y_{n}, y_{n-1}, y_{n-2}\right)^{T}$ and

$$
J_{F}\left(\bar{x}_{1}, \bar{y}_{1}\right)=\left(\begin{array}{cccccc}
0 & 2 & 0 & 0 & 0 & -\frac{1}{r} \\
1 & 0 & 0 & 0 & 0 & 0 \\
0 & 1 & 0 & 0 & 0 & 0 \\
0 & 0 & -\frac{1}{s} & 0 & 2 & 0 \\
0 & 0 & 0 & 1 & 0 & 0 \\
0 & 0 & 0 & 0 & 1 & 0
\end{array}\right) .
$$

The characteristic equation of $J_{F}\left(\bar{x}_{0}, \bar{y}_{0}\right)\left(\bar{x}_{1}, \bar{y}_{1}\right)$ is as follows:

$$
\lambda^{6}-4 \lambda^{4}+4 \lambda^{2}-\frac{1}{r s}=0 .
$$

From this characteristic equation, we obtain

$$
\Delta_{6 \times 6}=\left(\begin{array}{cccccc}
0 & 0 & 0 & 0 & 0 & 0 \\
1 & -4 & 4 & -\frac{1}{r s} & 0 & 0 \\
0 & 0 & 0 & 0 & 0 & 0 \\
0 & 1 & -4 & 4 & -\frac{1}{r s} & 0 \\
0 & 0 & 0 & 0 & 0 & 0 \\
0 & 0 & 1 & -4 & 4 & -\frac{1}{r s}
\end{array}\right) .
$$

It is clear that not all of $\Delta_{6 \times 6}>0$. Therefore, by Theorem 2 , the unique positive equilibrium point $\left(\bar{x}_{1}, \bar{y}_{1}\right)$ is unstable. But as the product of roots equals $-\frac{1}{r s}$, we conclude that there is at least one root with modulus less than one. Therefore, $\left(\bar{x}_{1}, \bar{y}_{1}\right)$ is a Saddle point.

This completes the proof.

\section{Global stability of the Zero equilibrium Point}

Consider the subsets $I_{j}, j \in\{1,2,3,4\}$ in $\mathbb{R}$, where

$$
\begin{aligned}
& \left.I_{1}=\right] 0, \bar{x}_{1}[\times] \bar{y}_{1}, \infty[; \\
& \left.I_{2}=\right] \bar{x}_{1}, \infty[\times] 0, \bar{y}_{1}[; \\
& \left.I_{3}=\right] 0, \bar{x}_{1}[\times] 0, \bar{y}_{1}[; \\
& \left.I_{4}=\right] \bar{x}_{1}, \infty[\times] \bar{y}_{1}, \infty[.
\end{aligned}
$$

Theorem 5. If rs $>1$, then the subsets $\left.I_{1}=\right] 0, \bar{x}_{1}[\times] \bar{y}_{1}, \infty\left[\right.$ and $I_{2}=$ ] $\bar{x}_{1}, \infty[\times] 0, \bar{y}_{1}\left[\right.$ are invariant subsets of $\mathbb{R}^{2}$ for system (3). 
Proof. We show that $I_{1}$ is an invariant subset of $\mathbb{R}^{2}$ for system (3). The proof is by induction on $n$.

Let $\left(x_{-i}, y_{-i}\right) \in I_{1}$ for $i \in\{0,1,2\}$. Then

$$
x_{1}=\frac{r x_{-1}^{2}}{1+y_{-2}}<\frac{r \bar{x}_{1}^{2}}{1+\bar{y}_{1}}=\bar{x}_{1}
$$

and

$$
y_{1}=\frac{s y_{-1}^{2}}{1+x_{-2}}>\frac{s \bar{y}_{1}^{2}}{1+\bar{x}_{1}}=\bar{y}_{1} .
$$

That is $\left(x_{1}, y_{1}\right) \in I_{1}$. Suppose that for a fixed $n \in \mathbb{N}$ we have $\left(x_{n-i}, y_{n-i}\right) \in$ $I_{1}$, for $i \in\{0,1,2\}$. Then

$$
x_{n+1}=\frac{r x_{n-1}^{2}}{1+y_{n-2}}<\frac{r \bar{x}_{1}^{2}}{1+\bar{y}_{1}}=\bar{x}_{1}
$$

and

$$
y_{n+1}=\frac{s y_{n-1}^{2}}{1+x_{n-2}}>\frac{s \bar{y}_{1}^{2}}{1+\bar{x}_{1}}=\bar{y}_{1} .
$$

That is $\left(x_{n+1}, y_{n+1}\right) \in I_{1}$. Therefore, $I_{1}$ is an invariant subset of $\mathbb{R}^{2}$ for system (3).

Similarly, we can show that $I_{2}$ is an invariant subset of $\mathbb{R}^{2}$ for system (3). This completes the proof.

We have shown that the zero equilibrium point is locally asymptotically stable. In the following results, we shall show that the zero equilibrium point is a global attractor with basin as well as it is globally asymptotically stable.

Theorem 6. Assume that $r<1$ and $s<1$ in system (3). Then the equilibrium point $\left(\bar{x}_{0}, \bar{y}_{0}\right)$ is a global attractor with basin $] 0,1\left[{ }^{2}\right.$.

Proof. Suppose that the initial points $\left.\left(x_{-i}, y_{-i}\right) \in\right] 0,1\left[^{2}, i \in\{0,1,2\}\right.$. It follows that

$$
x_{n+1}=\frac{r x_{n-1}^{2}}{1+y_{n-2}}<r x_{n-1}^{2}<r x_{n-1} .
$$

Then the subsequences $\left\{x_{2 n+i}\right\}_{n=-1}^{\infty}, i=1,2$ converges to zero and so the $x_{n} \rightarrow 0$ as $n \rightarrow \infty$.

Similarly $y_{n} \rightarrow 0$ as $n \rightarrow \infty$. Therefore $\left\{\left(x_{n}, y_{n}\right)\right\}_{n=-2}^{\infty}$ converges to the zero equilibrium point $(0,0)$ as $n \rightarrow \infty$. This completes the proof.

We put the following result without proof.

Theorem 7. Consider system (3) and let $x_{-i}, y_{-i}, i \in\{0,1,2\}$ be nonnegative real numbers. Then for all $n \geq 1$, we have

$$
x_{n} \leq \begin{cases}r^{2^{\frac{n-1}{2}}} x_{-1}^{2^{\frac{n+1}{2}}}, & n=1,3, \ldots \\ r^{2^{\left(\frac{n}{2}\right)-1}} x_{0}^{2^{\frac{n}{2}}}, & n=2,4, \ldots\end{cases}
$$


and

$$
y_{n} \leq \begin{cases}s^{2^{\frac{n-1}{2}}} y_{-1}^{2^{\frac{n+1}{2}}}, & n=1,3, \ldots \\ s^{2^{\left(\frac{n}{2}\right)-1}} y_{0}^{2^{\frac{n}{2}}}, & n=2,4, \ldots\end{cases}
$$

Theorem 8. Assume that $r<1$ and $s<1$. Then the equilibrium point $\left(\bar{x}_{0}, \bar{y}_{0}\right)$ of system (3) is globally asymptotically stable.

Proof. The proof is a direct consequence of Theorem 3 and Theorem 7 .

In the following result, we shall show that there exist unbounded solutions of system (3).

Theorem 9. Let $\left\{\left(x_{n}, y_{n}\right)\right\}_{n=-2}^{\infty}$ be a solution of system (3). If $r s>1$, then we have the following:

(1) If $\left(x_{-i}, y_{-i}\right) \in I_{3}, i \in\{0,2\}$ and $\left(x_{-1}, y_{-1}\right) \in I_{4}$, then the subsequence $\left\{\left(x_{2 n}, y_{2 n}\right)\right\}_{n=0}^{\infty}$ converges to the point $(0,0)$ and the subsequence $\left\{\left(x_{2 n+1}, y_{2 n+1}\right)\right\}_{n=0}^{\infty}$ diverges to $(\infty, \infty)$.

(2) If $\left(x_{-i}, y_{-i}\right) \in I_{4}, i \in\{0,2\}$ and $\left(x_{-1}, y_{-1}\right) \in I_{3}$, then the subsequence $\left\{\left(x_{2 n+1}, y_{2 n+1}\right)\right\}_{n=0}^{\infty}$ converges to the point $(0,0)$ and the subsequence $\left\{\left(x_{2 n}, y_{2 n}\right)\right\}_{n=0}^{\infty}$ diverges to $(\infty, \infty)$.

Proof. We shall prove (1). The proof of (2) is similar and will be omitted.

The condition $\left(x_{-i}, y_{-i}\right) \in I_{3}, i \in\{0,2\}$ and $\left(x_{-1}, y_{-1}\right) \in I_{4}$ is equivalent to

$$
x_{-2}, x_{0}<\bar{x}_{1}<x_{-1} \text { and } y_{-2}, y_{0}<\bar{y}_{1}<y_{-1} .
$$

Then we have

$$
x_{1}=\frac{r x_{-1}}{1+y_{-2}} x_{-1}>\frac{r \bar{x}_{1}}{1+\bar{y}_{1}} x_{-1}=x_{-1}>\bar{x}_{1}
$$

and

$$
x_{3}=\frac{r x_{1}}{1+y_{0}} x_{1}>\frac{r \bar{x}_{1}}{1+\bar{y}_{1}} x_{1}=x_{1}>x_{-1}>\bar{x}_{1} .
$$

Using induction on $n$, we can obtain

$$
\bar{x}_{1}<x_{-1}<\cdots<x_{2 n-1}<x_{2 n+1}<\cdots .
$$

Similarly,

$$
\bar{y}_{1}<y_{-1}<\cdots<y_{2 n-1}<y_{2 n+1}<\cdots .
$$

That is the subsequence $\left\{\left(x_{2 n+1}, y_{2 n+1}\right)\right\}_{n=0}^{\infty}$ diverges to $(\infty, \infty)$.

By the same argument, we can obtain

$$
\bar{x}_{1}>x_{0}>\cdots>x_{2 n-2}>x_{2 n}>\cdots,
$$

and

$$
\bar{y}_{1}>y_{0}>\cdots>y_{2 n-2}>y_{2 n}>\cdots .
$$

That is the subsequence $\left\{\left(x_{2 n}, y_{2 n}\right)\right\}_{n=0}^{\infty}$ converges to the point $(0,0)$. This completes the proof. 


\section{PERIODICITY}

In the following result, we show that under certain conditions, system (3) possesses period-2 solutions.

Theorem 10. Assume that $r s>1$ in system (3). Then we have the following:

(1) If $x_{-2}=x_{0}=0, x_{-1}=\frac{1}{r}\left(x_{-2}=x_{0}=\frac{1}{r}, x_{-1}=0\right)$ and $y_{-2}=$ $y_{-1}=y_{0}=0$, then system (3) possesses the period-2 solution

$$
\left\{\ldots,(0,0),\left(\frac{1}{r}, 0\right),(0,0),\left(\frac{1}{r}, 0\right), \ldots\right\} \text {. }
$$

(2) If $x_{-2}=x_{-1}=x_{0}=0$ and $y_{-2}=y_{0}=0, y_{-1}=\frac{1}{s} \quad\left(y_{-2}=y_{0}=\frac{1}{s}\right.$, $\left.y_{-1}=0\right)$, then system (3) possesses the period-2 solution

$$
\left\{\ldots,(0,0),\left(0, \frac{1}{s}\right),(0,0),\left(0, \frac{1}{s}\right), \ldots\right\} \text {. }
$$

(3) If $x_{-2}=x_{0}=0, x_{-1}=\bar{x}_{1}\left(x_{-2}=x_{0}=\bar{x}_{1}, x_{-1}=0\right)$ and $y_{-2}=$ $y_{0}=\bar{y}_{1}, y_{-1}=0\left(y_{-2}=y_{0}=0, y_{-1}=\bar{y}_{1}\right)$, then system (3) possesses the period-2 solution

$$
\left\{\ldots,\left(0, \bar{y}_{1}\right),\left(\bar{x}_{1}, 0\right),\left(0, \bar{y}_{1}\right),\left(\bar{x}_{1}, 0\right), \ldots\right\} .
$$

(4) If $x_{-2}=x_{0}=0, x_{-1}=\frac{1}{r}\left(x_{-2}=x_{0}=\frac{1}{r}, x_{-1}=0\right)$ and $y_{-2}=y_{0}=$ $0, y_{-1}=\frac{1}{s}\left(y_{-2}=y_{0}=\frac{1}{s}, y_{-1}=0\right)$, then system (3) possesses the period-2 solution

$$
\left\{\ldots,(0,0),\left(\frac{1}{r}, \frac{1}{s}\right),(0,0),\left(\frac{1}{r}, \frac{1}{s}\right), \ldots\right\} .
$$

(5) If $x_{-2}=x_{0}=0, x_{-1}=\bar{x}_{1}\left(x_{-2}=x_{0}=\bar{x}_{1}, x_{-1}=0\right)$ and $y_{-2}=$ $y_{0}=\bar{y}_{1}, y_{-1}=\frac{1}{s}\left(y_{-2}=y_{0}=\frac{1}{s}, y_{-1}=\bar{y}_{1}\right)$, then system (3) possesses the period-2 solution

$$
\left\{\ldots,\left(0, \bar{y}_{1}\right),\left(\bar{x}_{1}, \frac{1}{s}\right),\left(0, \bar{y}_{1}\right),\left(\bar{x}_{1}, \frac{1}{s}\right), \ldots\right\} .
$$

(6) If $x_{-2}=x_{0}=\frac{1}{r}, x_{-1}=\bar{x}_{1}\left(x_{-2}=x_{0}=\bar{x}_{1}, x_{-1}=\frac{1}{r}\right)$ and $y_{-2}=$ $y_{0}=\bar{y}_{1}, y_{-1}=0\left(y_{-2}=y_{0}=0, y_{-1}=\bar{y}_{1}\right)$, then system (3) possesses the period-2 solution

$$
\left\{\ldots,\left(\frac{1}{r}, \bar{y}_{1}\right),\left(\bar{x}_{1}, 0\right),\left(\frac{1}{r}, \bar{y}_{1}\right),\left(\bar{x}_{1}, 0\right), \ldots\right\} .
$$

Proof. Suppose that

$$
\left\{\left(p_{1}, q_{1}\right),\left(p_{2}, q_{2}\right),\left(p_{1}, q_{1}\right),\left(p_{2}, q_{2}\right), \ldots\right\}
$$

is a period-2 solution of system (3), where at least $p_{1} \neq p_{2}$ or $q_{1} \neq q_{2}$. Then we have

$$
\begin{aligned}
& p_{2}=\frac{r p_{2}^{2}}{1+q_{1}}, p_{1}=\frac{r p_{1}^{2}}{1+q_{2}}, \\
& q_{2}=\frac{s q_{2}^{2}}{1+p_{1}}, q_{1}=\frac{s q_{1}^{2}}{1+p_{2}} .
\end{aligned}
$$


If $p_{i}, q_{i}$ are positive real numbers for $i=1,2$, then after some calculations using (7) and (2) we can obtain $p_{1}=p_{2}=\bar{x}_{1}$ and $q_{1}=q_{2}=\bar{y}_{1}$ which is a contradiction of our assumption. This means that, period-2 solution of the form (7) with positive real numbers $p_{i}, q_{i}, i=1,2$ does not exist.

(1) Suppose that $y_{-2}=y_{-1}=y_{0}=0$. Then from system (3), we have

$$
x_{n+1}=r x_{n-1}^{2}, \quad y_{n}=0, \quad \text { for all } n \geq 0 .
$$

Using (7) and (8), we get

$$
q_{1}=q_{2}=0, \quad p_{1}=r p_{1}^{2} \quad \text { and } \quad p_{2}=r p_{2}^{2} .
$$

Taking into account that $p_{1} \neq p_{2}$, we get

$$
p_{1}=0, \quad p_{2}=\frac{1}{r} \quad \text { or } \quad p_{2}=0, \quad p_{1}=\frac{1}{r} .
$$

Therefore, if $x_{-2}=x_{0}=p_{1}=0, x_{-1}=p_{2}=\frac{1}{r}\left(x_{-2}=x_{0}=p_{2}=\frac{1}{r}\right.$, $\left.x_{-1}=p_{1}=0\right)$, we get the period- 2 solution

$$
\left\{\ldots,(0,0),\left(\frac{1}{r}, 0\right),(0,0),\left(\frac{1}{r}, 0\right), \ldots\right\} .
$$

(2) The proof is similar to (1) and will be omitted.

(3) Suppose that $p_{1}=q_{2}=0$. Using (7) and (8), one of the nonzero solutions for $q_{1}$ and $p_{2}$ is $q_{1}=\bar{y}_{1}$ and $p_{2}=\bar{x}_{1}$. Therefore, if $x_{-2}=x_{0}=$ $0, x_{-1}=p_{2}=\bar{x}_{1}\left(x_{-2}=x_{0}=p_{2}=\bar{x}_{1}, x_{-1}=0\right)$ and $y_{-2}=y_{0}=$ $q_{1}=\bar{y}_{1}, y_{-1}=0\left(y_{-2}=y_{0}=0, y_{-1}=q_{1}=\bar{y}_{1}\right)$, then system (3) possesses the period-2 solution $\left\{\ldots,\left(0, \bar{y}_{1}\right),\left(\bar{x}_{1}, 0\right),\left(0, \bar{y}_{1}\right),\left(\bar{x}_{1}, 0\right), \ldots\right\}$.

The proof of (4), (5) and (6) is similar and will be omitted.

This completes the proof.

\section{Rate of Convergence}

In this section, we shall study the rate of convergence of a solution that converges to the equilibrium point $(0,0)$ of the system (3). The following result gives the rate of convergence of the solution of a system of difference equations:

$$
X_{n+1}=[A+B(n)] X_{n},
$$

where $X_{n}$ is a six-dimensional vector, $A \in C^{6 \times 6}$ is a constant matrix and $B: \mathbb{Z}^{+} \rightarrow C^{6 \times 6}$ is a matrix function satisfying

$$
\|B(n)\| \rightarrow 0, \text { when } n \rightarrow \infty,
$$

where $\|$.$\| denotes any matrix norm which is associated with the vector norm.$ Also $\|\cdot\|$ denotes the Euclidean norm in $\mathbb{R}^{2}$ given by

$$
\|(x, y)\|=\sqrt{x^{2}+y^{2}} .
$$


Theorem 11 ([25]). Assume that condition (10) holds, if $X_{n}$ is a solution of (9), then either $X_{n}=0$ for all large $n$ or

$$
\theta=\lim _{n \rightarrow \infty} \sqrt[n]{\left\|X_{n}\right\|}
$$

exists and $\theta$ is equal to the modulus of one the eigenvalues of the matrix $A$.

Theorem 12 ([25]). Assume that condition (10) holds, if $X_{n}$ is a solution of (9), then either $X_{n}=0$ for all large $n$ or

$$
\theta=\lim _{n \rightarrow \infty} \frac{\left\|X_{n+1}\right\|}{\left\|X_{n}\right\|}
$$

exists and $\theta$ is equal to the modulus of one the eigenvalues of the matrix $A$.

Assume that $\lim _{n \rightarrow \infty} x_{n}=\bar{x}$ and $\lim _{n \rightarrow \infty} y_{n}=\bar{y}$ and consider the error vector

$$
E_{n}=\left(\begin{array}{c}
e_{n}^{1} \\
e_{n-1}^{1} \\
e_{n-2}^{1} \\
e_{n}^{2} \\
e_{n-1}^{2} \\
e_{n-2}^{2}
\end{array}\right)=\left(\begin{array}{c}
x_{n}-\bar{x} \\
x_{n-1}-\bar{x} \\
x_{n-2}-\bar{x} \\
y_{n}-\bar{y} \\
y_{n-1}-\bar{y} \\
y_{n-2}-\bar{y}
\end{array}\right) .
$$

We shall find a system satisfied by the error terms. The error terms satisfy the relations

$$
\begin{aligned}
& x_{n+1}-\bar{x}=\sum_{i=0}^{2} A_{i n}\left(x_{n-i}-\bar{x}\right)+\sum_{i=0}^{2} B_{i n}\left(y_{n-i}-\bar{y}\right), \\
& y_{n+1}-\bar{y}=\sum_{i=0}^{2} C_{i n}\left(x_{n-i}-\bar{x}\right)+\sum_{i=0}^{2} D_{i n}\left(y_{n-i}-\bar{y}\right) .
\end{aligned}
$$

where

$$
\begin{array}{llll}
A_{0 n}=0, & A_{1 n}=\frac{r\left(x_{n-1}+\bar{x}\right)}{1+\bar{y}}, & A_{2 n}=0, \\
B_{0 n}=0, \quad B_{1 n}=0, & B_{2 n}=-\frac{r x_{n-1}^{2}}{\left(1+y_{n-2}\right)(1+\bar{y})}, \\
C_{0 n}=0, \quad C_{1 n}=0, & C_{2 n}=-\frac{s y_{n-1}^{2}}{\left(1+x_{n-2}\right)(1+\bar{x})}, \\
D_{0 n}=0, \quad D_{1 n}=\frac{s\left(y_{n-1}+\bar{y}\right)}{1+\bar{x}}, & D_{2 n}=0 .
\end{array}
$$

We can write system (11) as

$$
\begin{aligned}
& e_{n+1}^{1}=\sum_{i=0}^{2} A_{i n} e_{n-i}^{1}+\sum_{i=0}^{2} B_{i n} e_{n-i}^{2}, \\
& e_{n+1}^{2}=\sum_{i=0}^{2} C_{i n} e_{n-i}^{1}+\sum_{i=0}^{2} D_{i n} e_{n-i}^{2} .
\end{aligned}
$$

Taking the limits of $A_{i n}, B_{i n}, C_{i n}, D_{i n}, i=0,1,2$ we obtain

$$
\lim _{n \rightarrow \infty} A_{\text {in }}=0 \text { for } i \in\{0,2\} \text { and } \lim _{n \rightarrow \infty} A_{1 n}=\frac{2 r \bar{x}}{1+\bar{y}},
$$




$$
\begin{aligned}
& \lim _{n \rightarrow \infty} B_{i n}=0 \text { for } i \in\{0,1\} \text { and } \lim _{n \rightarrow \infty} B_{2 n}=-\frac{r \bar{x}^{2}}{(1+\bar{y})^{2}}, \\
& \lim _{n \rightarrow \infty} C_{i n}=0 \text { for } i \in\{0,1\} \text { and } \lim _{n \rightarrow \infty} C_{2 n}=-\frac{s \bar{y}^{2}}{(1+\bar{x})^{2}}, \\
& \lim _{n \rightarrow \infty} D_{i n}=0 \text { for } i \in\{0,2\} \text { and } \lim _{n \rightarrow \infty} D_{1 n}=\frac{2 s \bar{y}}{1+\bar{x}} .
\end{aligned}
$$

That is we can write

$$
\begin{aligned}
& A_{1 n}=\frac{2 r \bar{x}}{1+\bar{y}}+a_{n}, B_{2 n}=-\frac{r \bar{x}^{2}}{(1+\bar{y})^{2}}+b_{n}, \\
& C_{2 n}=-\frac{s \bar{y}^{2}}{(1+\bar{x})^{2}}+c_{n}, D_{1 n}=\frac{2 s \bar{y}}{1+\bar{x}}+d_{n},
\end{aligned}
$$

where $a_{n} \rightarrow 0, b_{n} \rightarrow 0, c_{n} \rightarrow 0$ and $d_{n} \rightarrow 0$ as $n \rightarrow \infty$.

Then we can write

$$
E_{n+1}=\left[K+B_{n}\right] E_{n}
$$

where

$$
K=\left(\begin{array}{cccccc}
0 & \frac{2 r \bar{x}}{1+\bar{y}} & 0 & 0 & 0 & -\frac{r \bar{x}^{2}}{(1+\bar{y})^{2}} \\
1 & 0 & 0 & 0 & 0 & 0 \\
0 & 1 & 0 & 0 & 0 & 0 \\
0 & 0 & -\frac{s \bar{y}^{2}}{(1+\bar{x})^{2}} & 0 & \frac{2 s \bar{y}}{1+\bar{x}} & 0 \\
0 & 0 & 0 & 1 & 0 & 0 \\
0 & 0 & 0 & 0 & 1 & 0
\end{array}\right)
$$

and

$$
B_{n}=\left(\begin{array}{cccccc}
0 & a_{n} & 0 & 0 & 0 & b_{n} \\
0 & 0 & 0 & 0 & 0 & 0 \\
0 & 0 & 0 & 0 & 0 & 0 \\
0 & 0 & c_{n} & 0 & d_{n} & 0 \\
0 & 0 & 0 & 0 & 0 & 0 \\
0 & 0 & 0 & 0 & 0 & 0
\end{array}\right)
$$

with $\left\|B_{n}\right\| \rightarrow 0$ as $n \rightarrow \infty$.

Thus, the limitting system of error terms about an equilibrium point $(\bar{x}, \bar{y})$ can be written

$$
\left(\begin{array}{c}
e_{n+1}^{1} \\
e_{n}^{1} \\
e_{n-1}^{1} \\
e_{n+1}^{2} \\
e_{n}^{2} \\
e_{n-1}^{2}
\end{array}\right)=\left(\begin{array}{cccccc}
0 & \frac{2 r \bar{x}}{1+\bar{y}} & 0 & 0 & 0 & -\frac{r \bar{x}^{2}}{(1+\bar{y})^{2}} \\
1 & 0 & 0 & 0 & 0 & 0 \\
0 & 1 & 0 & 0 & 0 & 0 \\
0 & 0 & -\frac{s \bar{y}^{2}}{(1+\bar{x})^{2}} & 0 & \frac{2 s \bar{y}}{1+\bar{x}} & 0 \\
0 & 0 & 0 & 1 & 0 & 0 \\
0 & 0 & 0 & 0 & 1 & 0
\end{array}\right)\left(\begin{array}{c}
e_{n}^{1} \\
e_{n-1}^{1} \\
e_{n-2}^{1} \\
e_{n}^{2} \\
e_{n-1}^{2} \\
e_{n-2}^{2}
\end{array}\right)
$$

System (6) is similar to the linearized system of system (3) about an equilibrium point $(\bar{x}, \bar{y})$. 
System (6) about the equilibrium point $(0,0)$ can be written as

$$
\left(\begin{array}{c}
e_{n+1}^{1} \\
e_{n}^{1} \\
e_{n-1}^{1} \\
e_{n+1}^{2} \\
e_{n}^{2} \\
e_{n-1}^{2}
\end{array}\right)=\left(\begin{array}{llllll}
0 & 0 & 0 & 0 & 0 & 0 \\
1 & 0 & 0 & 0 & 0 & 0 \\
0 & 1 & 0 & 0 & 0 & 0 \\
0 & 0 & 0 & 0 & 0 & 0 \\
0 & 0 & 0 & 1 & 0 & 0 \\
0 & 0 & 0 & 0 & 1 & 0
\end{array}\right)\left(\begin{array}{c}
e_{n}^{1} \\
e_{n-1}^{1} \\
e_{n-2}^{1} \\
e_{n}^{2} \\
e_{n-1}^{2} \\
e_{n-2}^{2}
\end{array}\right)
$$

The matrix $K$ is the same as the Jacobian matrix evaluated at the zero equilibrium point. Using Theorems 11 and 12, we have the following result.

Corollary 1. Let $\left\{\left(x_{n}, y_{n}\right)\right\}_{n=-2}^{\infty}$ be a solution of the system (3) such that

$$
\lim _{n \rightarrow \infty} x_{n}=0 \text { and } \lim _{n \rightarrow \infty} y_{n}=0 \text {. }
$$

Then the error vector

$$
E_{n}=\left(\begin{array}{c}
e_{n}^{1} \\
e_{n-1}^{1} \\
e_{n-2}^{1} \\
e_{n}^{2} \\
e_{n-1}^{2} \\
e_{n-2}^{2}
\end{array}\right)=\left(\begin{array}{c}
x_{n} \\
x_{n-1} \\
x_{n-2} \\
y_{n} \\
y_{n-1} \\
y_{n-2}
\end{array}\right)
$$

of the solution $\left\{\left(x_{n}, y_{n}\right)\right\}_{n=-2}^{\infty}$ of the system (3) satisfies both of the following asymptotic relations:

$$
\lim _{n \rightarrow \infty} \sqrt[n]{\left\|E_{n}\right\|}=\sqrt{\sum_{i=0}^{2} x_{n-i}^{2}+\sum_{i=0}^{2} y_{n-i}^{2}}=0
$$

and

$$
\lim _{n \rightarrow \infty} \frac{\left\|E_{n+1}\right\|}{\left\|E_{n}\right\|}=\frac{\sqrt{\sum_{i=0}^{2} x_{n+1-i}^{2}+\sum_{i=0}^{2} y_{n+1-i}^{2}}}{\sqrt{\sum_{i=0}^{2} x_{n-i}^{2}+\sum_{i=0}^{2} y_{n-i}^{2}}}=0,
$$

where the eigenvalues of the Jacobian matrix evaluated at the equilibrium point $(0,0)$ are all equal to zero.

\section{Numerical SIMUlations}

In order to verify our theoretical results we consider several interesting numerical examples in this section. These examples represent different types of qualitative behavior of solutions of the system (3). All plots in this section are drawn with Mathematica.

Example 1. Figure 1. shows that if $r=0.7$ and $s=0.5(r<1$ and $s<1$ ), then a solution $\left\{\left(x_{n}, y_{n}\right)\right\}_{n=-2}^{\infty}$ of system (3) with initial conditions $x_{-2}=2.3, x_{-1}=1.3, x_{0}=1.5, y_{-2}=1.2, y_{-1}=0.5$ and $y_{0}=2$ converges to the zero equilibrium point $(0,0)$. 


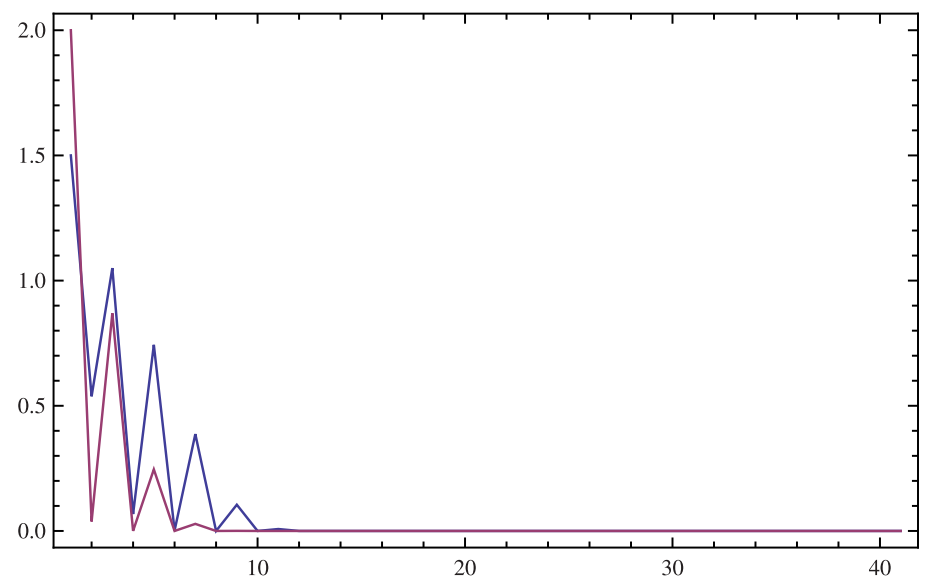

FIGURE 1. $x_{n+1}=\frac{0.7 x_{n-1}^{2}}{1+y_{n-2}}, y_{n+1}=\frac{0.5 y_{n-1}^{2}}{1+x_{n-2}}$

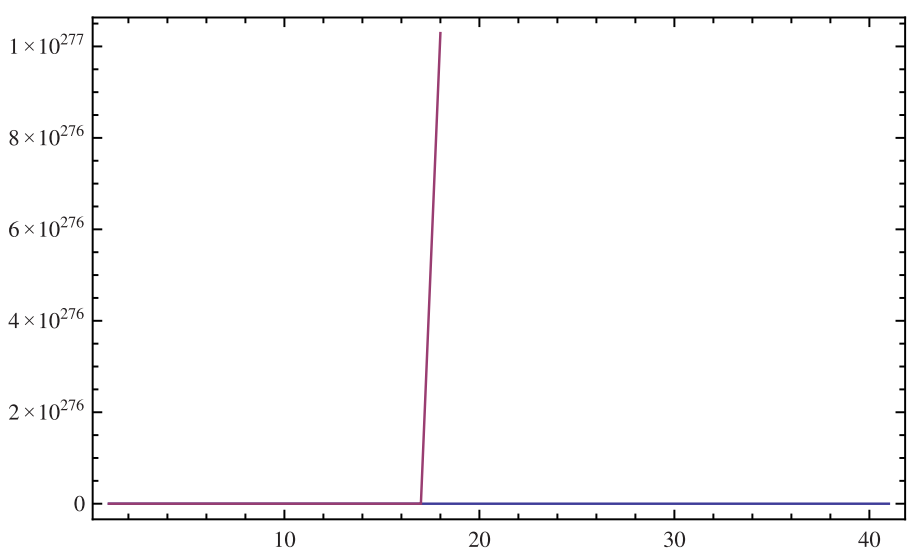

FIGURE 2. $x_{n+1}=\frac{2 x_{n-1}^{2}}{1+y_{n-2}}, y_{n+1}=\frac{3 y_{n-1}^{2}}{1+x_{n-2}}$

Example 2. Figure 2. shows that if $r=2$ and $s=3$ (rs $>1$ and $(\bar{x}, \bar{y})=(0.8,0.6))$, then a solution $\left\{\left(x_{n}, y_{n}\right)\right\}_{n=-2}^{\infty}$ of system (3) with initial conditions $x_{-2}=0.3, x_{-1}=0.3, x_{0}=0.5, y_{-2}=1.2, y_{-1}=1.5$ and $y_{0}=2$ $\left.\left.\left(\left(x_{-i}, y_{-i}\right) \in I_{1}=\right] 0,0.8\right] \times\right] 0.6, \infty[$, where $i \in\{0,1,2\})$ stay in the same subset $I_{1}$.

Example 3. Figure 3. shows that if $r=0.8$ and $s=1.3(r s=1.04>1)$, then a solution $\left\{\left(x_{n}, y_{n}\right)\right\}_{n=-2}^{\infty}$ of system (3) with initial conditions $x_{-2}=$ $x_{0}=\frac{1}{0.8}=1.25, x_{-1}=0, y_{-2}=y_{0}=0$ and $y_{-1}=0$ is periodic with prime period-2. The solution is of the form

$$
\{(1.25,0),(0,0),(1.25,0),(0,0), \ldots\} \text {. }
$$




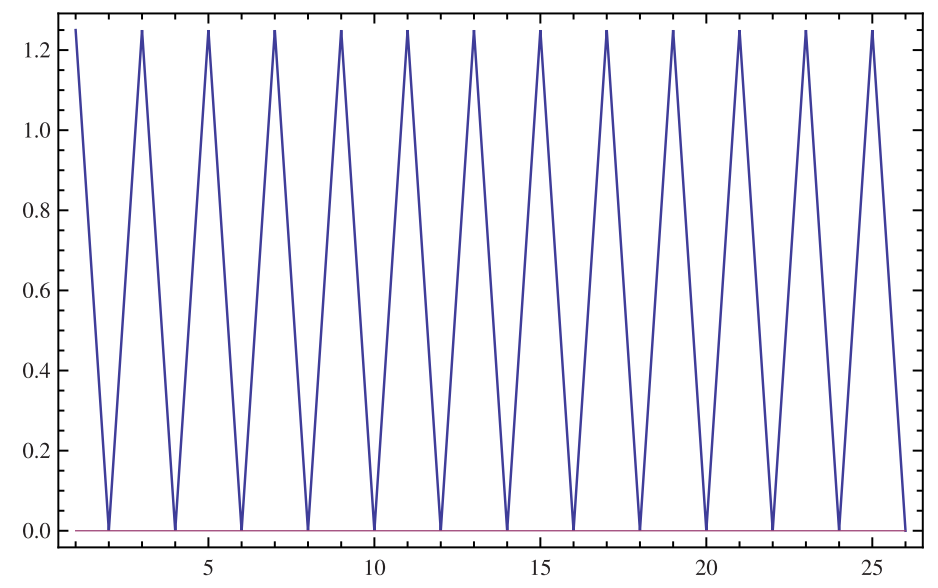

FIGURE 3. $x_{n+1}=\frac{0.8 x_{n-1}^{2}}{1+y_{n-2}}, y_{n+1}=\frac{1.3 y_{n-1}^{2}}{1+x_{n-2}}$

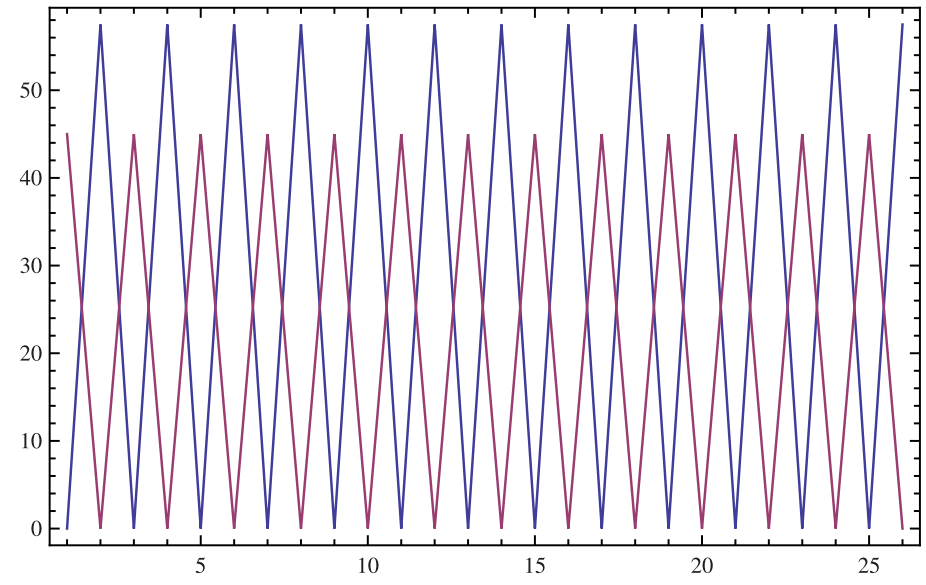

FigURE 4. $x_{n+1}=\frac{0.8 x_{n-1}^{2}}{1+y_{n-2}}, y_{n+1}=\frac{1.3 y_{n-1}^{2}}{1+x_{n-2}}$

Example 4. Figure 4. shows that if $r=0.8$ and $s=1.3(r s=1.04>1)$, then a solution $\left\{\left(x_{n}, y_{n}\right)\right\}_{n=-2}^{\infty}$ of system (3) with initial conditions $x_{-2}=$ $x_{0}=0, x_{-1}=57.5, y_{-2}=y_{0}=45$ and $y_{-1}=0$ is periodic with prime period-2. In fact the solution is

$$
\{(0,45),(57.5,0),(0,45),(57.5,0), \ldots\} \text {. }
$$

\section{Conclusion And SOME OPEN PROBlems}

In this paper, some properties of a higher dimensional difference system of equations were studied. Namely, we investigated the equilibria of the system (3) in details. Also, we investigated the stability character of these points 
using the linearization method . The main goal of dynamical systems theory is to approach the global behavior and the rate of convergence. Therefore, here we studied the global asymptotic stability and the rate of convergence of the zero equilibrium point of the system. Also, the existence of unbounded solutions and the periodicity of solutions of the system were studied. Even if it will be possible to obtain analytical results, it would be quite difficult to deal with them. So, numerical simulations were used to verify the correctness of analytical results.

Finally, we conjecture that our study can be extended to a system with higher order. We shall give some interesting open problems for difference systems of equations.

We conjecture that the obtained results are satisfied to the system

$$
x_{n+1}=\frac{\alpha x_{n-2 k-1}^{2}}{1+y_{n-2 l}}, y_{n+1}=\frac{\beta y_{n-2 k-1}^{2}}{1+x_{n-2 l}}, n=0,1, \ldots,
$$

where the parameters $\alpha, \beta$ are non-negative real numbers, the initial conditions $x_{-i}, y_{-i}$ are non-negative real numbers, $i=0,1, \ldots, \max \{2 l, 2 k+1\}$ and with natural numbers $l \geq 1, k \geq 1$.

Open Problem 1. Investigate the global behavior of the system of difference equations

$$
x_{n+1}=\frac{\alpha x_{n-1}^{p}}{1+y_{n-2}}, y_{n+1}=\frac{\beta y_{n-1}^{p}}{1+x_{n-2}}, n=0,1, \ldots,
$$

where the parameters $\alpha, \beta$ are non-negative real numbers, the initial conditions $x_{-i}, y_{-i}$ are non-negative real numbers, $i=0,1,2$ and $p$ is a positive integer.

Open Problem 2. Investigate the global behavior of the system of difference equations

$$
x_{n+1}=\frac{\alpha_{n} x_{n-1}^{2}}{1+y_{n-2}}, y_{n+1}=\frac{\beta_{n} y_{n-1}^{2}}{1+x_{n-2}}, n=0,1, \ldots,
$$

where $\alpha_{n}, \beta_{n}$ are sequences (these sequences can be chosen as convergent, periodic or bounded), the initial conditions $x_{-i}, y_{-i}$ are non-negative real numbers for $i=0,1,2$.

\section{REFERENCES}

[1] R. Abo-Zeid, Forbidden sets and stability in some rational difference equations, J. Difference Equ. Appl., 24 (2) (2018), 220-239.

[2] H. Bao, Dynamical behavior of a system of second-order nonlinear difference equations, Int. J. Differ. Equ., Volume 2015, Article ID: 679017, 7 pages.

[3] A. Brett, M. R. S. Kulenović, Basins of attraction for two-species competitive model with quadratic terms and the singular allee effect, Discrete Dyn. Nat. Soc., Volume 2015, Article ID: 847360, 16 pages. 
[4] Q. Din, T. F. Ibrahim, A. Q. Khan, Behavior of a competitive system of second-order difference equations, Sci. World J., Volume 2014, Article ID: 283982, 9 pages.

[5] Q. Din, E. M. Elsayed, Stability analysis of a discrete ecological model, Comput. Ecol. Softw., 4 (2) (2014), 89-103.

[6] H. M. El-Owaidy, A. M. Youssef, A. M. Ahmed, On the Dynamics of $x_{n+1}=$ $\left(b x_{n-1}^{2}\right)\left(A+B x_{n-2}\right)^{-1}$, Rostock. Math. Kolloq., 59 (2005), 11-18.

[7] E. M. Elsayed, Solutions of rational difference systems of order two, Math. Comput. Model., 55 (3) (2012), 378-384.

[8] E. M. Elsayed, H. El-Metwally, On the solutions of some nonlinear systems of difference equations, Adv. Difference Equ. 2013, 161 (2013).

[9] E. M. Elsayed, Solution for systems of difference equations of rational form of order two, Comput. Appl. Math., 33 (3) (2014), 751-765.

[10] E. M. Elsayed, A. M. Ahmed, Dynamics of a three-dimensional systems of rational difference equations, Math. Meth. Appl. Sci., 39 (5)(2016), 1026-1038.

[11] M. Göcen, A. Cebeci, On the periodic solutions of some systems of higher order difference equations, Rocky Mountain J. Math., 48 (3) (2018), 845-858.

[12] M. Göcen, M. Güneysu, The global attractivity of some rational difference equations, J. Comput. Anal. Appl., 25 (7) (2018), 1233-1243.

[13] M. Gümüs, The global asymptotic stability of a system of difference equations, J. Difference Equ. Appl., 24 (6) (2018), 976-991.

[14] M. Gümüş, Ö. Öcalan, The qualitative analysis of a rational system of diffrence equations, J. Fract. Calc. Appl., 9 (2) (2018), 113-126.

[15] T. F. Ibrahim, Closed form solution of a symmetric competitive system of rational difference equations, Stud. Math. Sci., 5 (1) (2012), 49-57.

[16] T. F. Ibrahim, Two-dimensional fractional system of nonlinear difference equations in the modeling competitive populations, Int. J. Bas. \& Appl. Sci., 12 (5) (2012), 103-121.

[17] L. X. Hu, X. M. Jia, Global asymptotic stability of a rational system, Abs. Appl. Anal., Volume 2014, Article ID: 286375, 6 pages.

[18] A. Q. Khan, M. N. Qureshi, Q. Din, Global dynamics of some systems of higher-order rational difference equations, Adv. Difference Equ. 2013, 354 (2013).

[19] V. Kocic, G. Ladas, Global Behavior of Nonlinear Difference Equations of Higher Order with Applications, Kluwer Academic Publishers, Dordrecht, 1993.

[20] M. R. S. Kulenović, G. Ladas, Dynamics of Second Order Rational Difference Equations: With Open Problems and Conjectures, Chapman \& Hall/CRC, 2001.

[21] M. R. S. Kulenović, O. Merino, Discrete Dynamical Systems and Difference Equations with Mathematica, Chapman \& Hall/CRC, Boca Raton, 2002.

[22] M. R. S. Kulenović, M. Pilling, Global dynamics of a certain two-dimensional competitive system of rational difference equations with quadratic terms, J. Comput. Anal. Appl., 19 (1) (2015), 156-166. 
[23] A. S. Kurbanl, C. Çinar, I. Yalçinkaya, On the behavior of positive solutions of the system of rational difference equations, Math. Comput. Model., 53 (5) (2011), 1261-1267.

[24] A. S. Kurbanl, On the behavior of solutions of the system of rational difference equations, Adv. Difference Equ. 2011, 40 (2011).

[25] M. Pituk, More on Poincaré's and perron's theorems for difference equations, J. Difference Equ. Appl., 8 (3) (2002), 201-216.

[26] B. Sroysang, Dynamics of a system of rational higher-order difference equation, Discrete Dyn. Nat. Soc., Volume 2013, Article ID: 179401, 5 pages.

[27] V. V. Khuong, M. N. Phong, On the global asymptotic stability of a second-order system of difference equations, South Asian J. Math., 4 (2) (2014), 93-103.

\section{MeHMET GÜMÜŞ}

Zonguldak BÜlent ECEVit University

FACUlty of Science and Arts

Department of Mathematics

67100, ZONGULDAK

TURKEY

E-mail address: m.gumus@beun.edu.tr

\section{RAAFAT ABo-Zeid}

The Higher Institute For

Engineering \& Technology Al-Obour

Department of Basic Science

Cairo

Egypt

E-mail address: abuzead73@yahoo.com 